\title{
Ewing's sarcoma/PNET of the Labia Majora an unusual presentation in 2 year old girl
}

\begin{abstract}
ES/PNET is a high-grade malignant neoplasm that often develops in the skeletal system. Primary extra skeletal ES/PNET is uncommon condition that rarely affects the female genital tract. Reports on the Ewing family of tumors involving the vulva/labia majora are extremely rare in the relevant literature. A 2 year old girl presented to the clinic with 6 months history of a right vulvar mass. She was admitted to our hospital for the vulvar mass, originally thought to be an inguinal hernia. The mass was excised under general anesthesia.

It was subsequently found to consist of small round cells positive for anti-CD99 antibody, thus suggesting a diagnosis of ES/PNET. Re-resection was performed five months later to obtain negative microscopic margins.

Our findings underline the crucial role of molecular biology techniques in the differential diagnosis of small round cell tumors in this unusual location. In summary, we describe a rare case of extra skeletal ES/PNET with distinct rosette-like structures in a 2 year old girl with a good prognosis.
\end{abstract}

Keywords: soft tissue-Ewing sarcoma, clinical characteristics, Labia majora
Volume 5 Issue 4 - 2016

Irena M, Suhih DJ, Majic V, Bajmak A

Pediatric Surgery Department, Institute for Children Diseases, Clinical Center of Montenegro

Correspondence: Irena Maric, Department of pediatric surgery Institute for Children Diseases, Clinical Center of Montenegro, Dalmatinska, Tel 69305489

Emailimaric@t-com.me

Received: March 27, 2016 | Published: October 18, 2016

\section{Introduction}

Ewing sarcoma represents approximately ten percent of the primary bone tumors and one percent of soft tissue tumors. Reports on the Ewing family of tumors involving the vulva/labia majora are extremely rare in the relevant literature. Only a few cases of vulva ES/ PNET have so far been reported.

The patient had localized disease treated with wide local excision and after surgery the patient received nine cycles of polychemotherapy. To our knowledge, this is the first reported case of extra skeletal Ewing's sarcoma confirmed with immunocytochemistry and genetic analysis in our country. Histological features include small round hyperchromatic and little mitotic activity. ${ }^{1-8}$ Because these findings are non-specific, immunohistochemistry is frequently. In more than $90 \%$ of cases, EWS cells express the adhesion receptor CD99. However, CD99 is not a specific marker for EWS as it is often also expressed in lymphoid cells of many normal tissues.

We present the first documented case of soft tissue Ewing sarcoma in our country. She is well after 1 year of follow-up.

\section{Case report}

A 2 year old girl was presented to the clinic with 6 months history of a right vulvar mass. On this admission, the patient 's general physical examination revealed a fixed mass and bulging tumor about $5 \mathrm{~cm}$ in size in the right inguinal region, originally thought to be an inguinal hernia. Parents provided information that the girl was in good condition without symptoms, only recently decreased appetite. Parents informed us that they noticed the mass was smaller during night.

The standard patient evaluation included also history examination, complete blood count, serum chemistries and ultrasound which showed the mass corresponds with visible intestine with paravesical hernial gate. Physical examination revealed no palpabile inguinal lymph node.
After having performed all initial examination, we decided to go for surgery. The patient had localized disease treated with local excision. The mass was excised under general anesthesia which was about $7 \mathrm{~cm}$ in size with no findings of nearby lymph node, no sign of herniation, inguinal canal intact, verified the tumorous mass in the region of the labia majora was not in contact with the surrounding structures .The patient's tumor markers, including CEA, AFP, beta HCG, CA125, were regularly tested and within normal limits. Histologically it was subsequently found to consist of small round cells positive for antiCD99 antibody, thus suggesting a diagnosis of Small round blue cell tumor-Alveolar botryoid rhabdomyosarcoma gr II (Figure 1).

After five cycles of poly-chemotherapy re-resection was performed to obtain negative microscopic margins and histological reexamination. Labia majora was excised subtotal and reconstructed. Pathology material was examined by a pathologist who had special expertise in sarcomas. The histopathological diagnosis was Ewing sarcoma/PNET. There was prominent mitotic activity, necrosis and hemorrhage. The diagnosis in our patient was revised from Alveolar botryoid rhabdomyosarcoma gr II to Ewing sarcoma/PNET, based on the results of immunohistochemical staining. The patient received postoperative another four cycles of polychemotherapy, CWS2009 protocol. Age of diagnosis, gender, lactate dehydrogenase level (LDH), primary tumor size, tumor size, no metastasis at presentation, response to induction chemotherapy, number of chemotherapy cycles, local treatment modality and resection margin were included in treatment and evaluation of our patient. We regularly followed up her hemogram, liver function and chest $\mathrm{X}$ ray.

MRI abdominal/pelvis performed 16 months after the operation showed neither residual tumor nor recurrent tumor. Chest CT also performed with normal findings. The patient was discharged without any complications (Figure 2).

Computerized tomography (CT) abdomen and pelvis was indicated as well as chest X ray and the results have shown the negative findings. After pediatric-oncology council, the child was followed by five courses of chemotherapy consisting of Holoxan, 
Oncovin and Adriamycin. During the chemotherapy, the child was repeatedly hospitalized for neutropenia and received granulocytic growth factor. MR pelvis showed postoperative sequelae differentiation in subcutaneous adipose tissue but without separation of macroscopically visible local recurrence. To our knowledge, this is the first reported case of extra skeletal Ewing's sarcoma confirmed with immunocytochemistry and genetic analysis in our country.

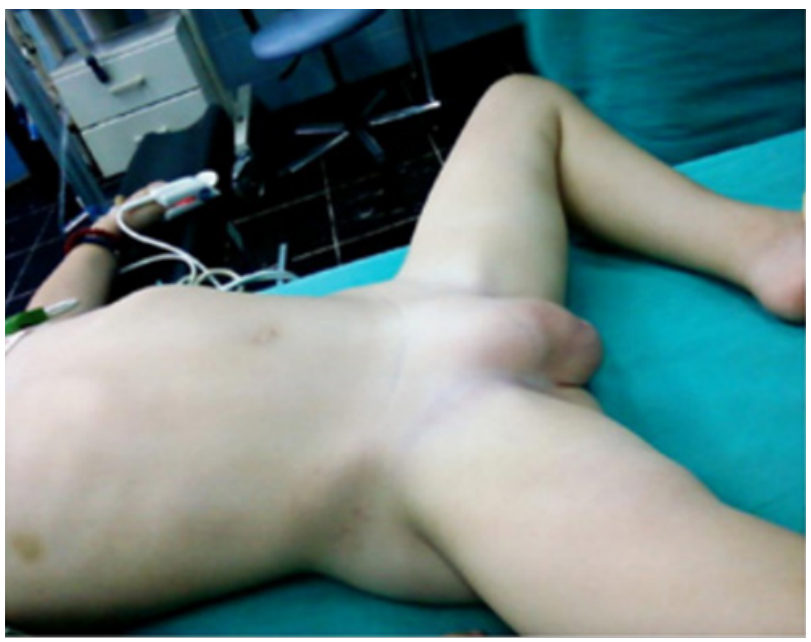

Figure I Right vulvar mass thought to be an inguinal hernia.

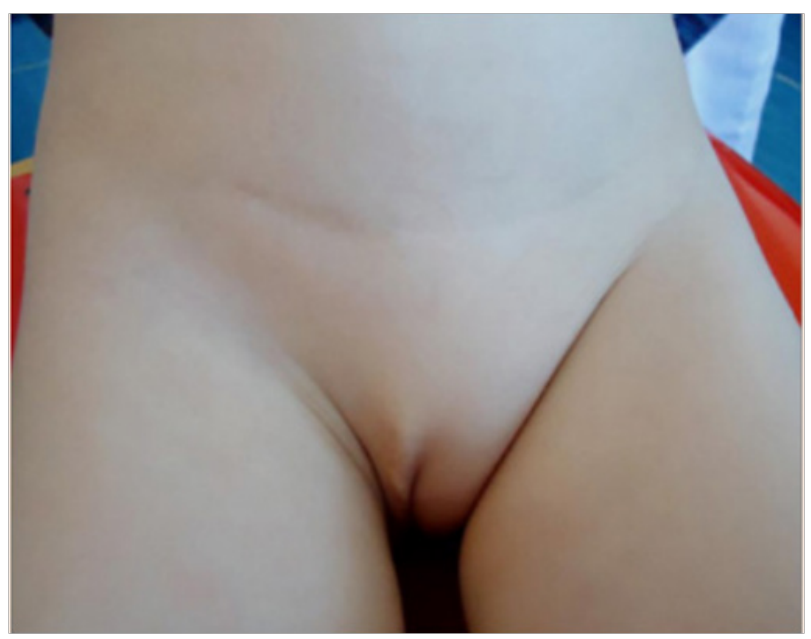

Figure 2 Current state after surgical reimpairment.

\section{Discussion}

The diagnosis of Ewing's sarcoma/PNET is based on histological, immunohistochemical and ultra-structural features. Ewing sarcoma/ PNET is now defined as a group of small round-cell sarcoma that shows varying degree of neuroectodermal differentiation. Extraosseous Ewing sarcomas can arise in the soft tissues of the chest wall, extremities, paravertebral and retroperitoneal regions, pelvis and abdomen, skin, head and neck, visceral organs but rarely occur in the female genital tract. Histological features include small round hyperchromatic and little mitotic activity. ${ }^{3,8}$ Because these findings are non-specific, immunohistochemistry is frequently (Figure 3 ).

Immunohistochemical markers currently used in the diagnosis of Ewing's sarcoma/PNET family of tumors include CD99, vimentin, desmin, neurofilament protein and neuron specific enolase. Combined treatment with surgical resection, chemotherapy resulted in a favorable outcome. Follow-up for these patients is very important, as the main aim to detect recurrent disease at a time when treatment can favorably influence the outcome for that patient. For sarcoma patients enrolled in clinical trials, there will usually be a standard follow-up regimen suggested by the trial protocol. The basic minimum followup would include careful clinical examination and a chest X-ray at regular intervals. Our patient is well after 1 year of follow-up.

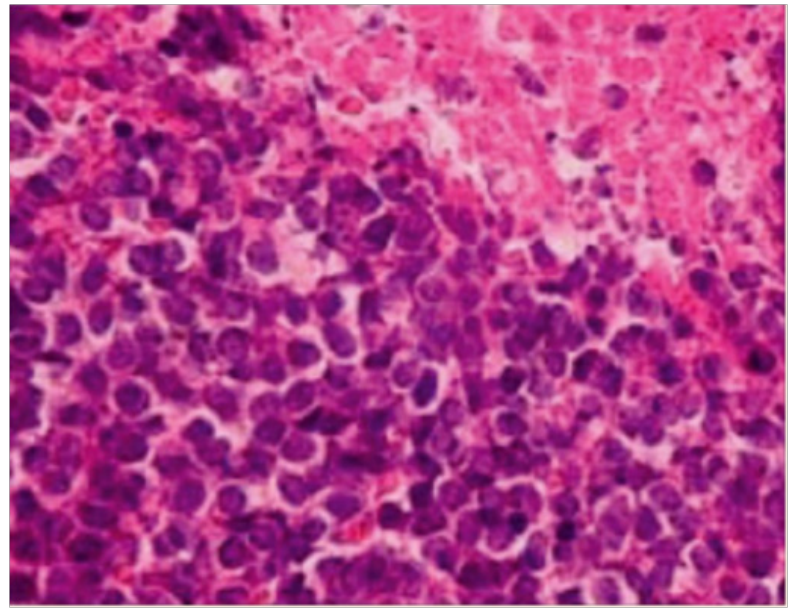

Figure 3 Ewing's sarcoma/PNET of labia majora - pathohistological finding of small round cell tumor.

\section{Conclusion}

With improvement in anticancer treatment the survival rate of cancer patients is increasing. Adequate surgical resection, aggressive chemotherapy and radiotherapy if indicated are the recommended therapy for patients with extra skeletal Ewing's sarcoma. In summary, we describe a rare case of extra skeletal ES/PNET with distinct rosettelike structures in a 2 year old girl with a good prognosis. Reports on the Ewing family of tumors involving the vulva/labia majora are extremely rare in the relevant literature. To our knowledge, this is the first reported case of extra skeletal Ewing's sarcoma confirmed with immunocytochemistry and genetic analysis in our country.

\section{Acknowledgments}

None.

\section{Conflicts of interest}

Authors declare that there is no conflict of interest.

\section{References}

1. LiaoX,XinX,LuX.Primary Ewing'ssarcoma-primitiveneuroectodermal tumor of the vagina. Gynecol Oncol. 2004;92(2):684-688.

2. Gaona-Luviano P, Unda-Franco E, González-Jara L, et al. Romero P,Medina-Franco H. Primitive neuroectodermal tumor of the vagina. Gynecol Oncol. 2003;91(2):456-458.

3. de Alava E, Gerald WL. Molecular biology of the Ewing's sarcoma/primitive neuroectodermal tumor family. $J$ Clin Oncol. 2000;18(1):204-213.

4. Smeland S, Wiebe T, Brosjö O, et al. Chemotherapy in Ewing's sarcoma. Acta Orthop Scand. 2004;75(311):87-91.

5. Granowetter L, West DC. The Ewing's sarcoma family tumors: Ewing's sarcoma and peripheral neuroectodermal tumor of bone and soft tissue. Rewiew. Cancer Treat Res. 1997;92:253-308. 
6. Zagar TM, Triche TJ, Kinsella TJ. Extraosseous Ewing's sarcoma: 25 years later. J Clin Oncol. 2008;26(26):4230-4232.

7. Yamada K, Sugiura H, Takahashi M. Single center experience of treatment of Ewing's family of tumors in Japan. J Orthop Sci. 2006;11(1):34-41.
8. Park JY, Lee S, Kang HJ, et al. Primary Ewing's sarcoma-primitive neuroectodermal tumor of the uterus: a case report and literature review. Gynecol Oncol. 2007;106(2):427-432. 